\title{
Magic Room: A Smart Space for Children with Neurodevelopmental Disorder
}

\author{
Franca Garzotto and Mirko Gelsomini \\ Politecnico di Milano, Italy
}

\begin{abstract}
We exploit recent advances in Cyber-Physical Systems (CPSs) technology to create the "Magic Room", a smart space created for use by children with Neurodevelopmental Disorder (NDD) and their caregivers. The Magic Room has been designed in cooperation with NDD specialists and supports multimodal embodied interaction by providing controllable stimuli to the vestibular, proprioceptive, and tactile sensory systems through various smart devices, e.g., ambient projections, physical objects, toys, ambient and mobile lights, aromas, and soap bubbles. We have deployed the Magic Room at two therapeutic centers. We performed exploratory studies with eight caregivers and nineteen children with severe impairments in the NDD spectrum, who used the smart room across four months with encouraging results. Our work may pave the ground for new research in the arena of CPSs for subjects with NDD and inspire new interventions for this target group.
\end{abstract}

\section{Author Keywords}

Neurodevelopmental Disorder, Children, Embodied interaction, Smart Space, Cyber-Physical System, Multi-Sensory Environment, Snoezelen

\section{INTRODUCTION}

Cyber-Physical Systems (CPSs) are "smart" systems made up of interacting networks of physical and computational components. As the power and popularity of these technologies increase, we face the challenge of making them accessible to and useful for people with disability. We address this issue in the domain of Neurodevelopmental Disorders (NDD). NDD is an umbrella term comprising a group of disorders (e.g., Intellectual Disability, Attention Deficit Hyperactivity Disorder, and ASD - Autism Spectrum Disorder) that arise during the developmental period and are associated to deficits in the cognitive, social, and motor spheres, leading to severe impairments in adaptive behavior and basic life skills.

We exploit CPS technology to transform a regular room into a multisensory multimodal interactive environment called Magic Room. The Magic Room aims at providing a pleasurable experiential space for children with NDD to learn-through-play, helping them to exercise the perceptual system and practice social, emotional, cognitive and motor skills. Our smart space supports multimodal embodied interaction (based on touch, manipulation, gestures, movements, and voice) with ambient projections, physical objects, and lights, and offers stimuli to the vestibular, proprioceptive and tactile sensory systems. Stimuli and interactions can be digitally controlled on-the-fly by caregivers and customized to the needs of each single child or children's group.

Our work's main contribution is to provide evidence of the deployment of a real smart multisensory space's designed in collaboration with NDD specialists having children with severe impairments in mind. The Magic Room is still in use at the center in Milan that collaborated with its design and evaluation. A second installation has been recently deployed at a large center for autism in Rome, and its integration in regular interventions will start after the summer break. The paper also offers insights, emerged from exploratory studies with nineteen children with NDD, on how this target groups might behave in the Magic Room, which may inform the design of future smart spaces.

\section{RELATED WORK}

For the past seven years, we have been collaborating with special schools, care centers and therapeutic institutions in Italy and abroad to design, develop and evaluate interactive technologies for children with NDD. From a methodological and technological perspective, the Magic Room integrates and extends our previous experiences. It is also inspired by other researchers' projects as well as existing multisensory practices for subjects with NDD.

The Magic Room's rationale is grounded on the theories of embodied cognition [12] and sensory integration [8]. The former emphasizes the formative role of embodiment (the way an organism's sensorimotor capacities enable it to successfully interact with the physical environment) in the development of cognitive skills. Embodied experiences promote cognitive processes linked to mastering sensorimotor contingencies and stimulate higher-level cognitive skills such as mental imagery, working and implicit memory, reasoning and problem solving. Sensory integration theories posit that learning is dependent upon the ability to take, integrate, and process multiple sensory information in order to plan and organize behavior. When this process 
has deficits, as it is thought to happen in subjects with NDD, an abnormal mental representation of the external world is created, which in turn affects the capability of appropriate behaviors in all aspects of life. Specific interventions for persons with NDD aim at stimulating basic perceptual mechanisms and promoting perceptual learning [4]. They often take place in a dedicated Multi-Sensory Environment (oftentimes referred to as Snoezelen ${ }^{\mathrm{R}}$ [6]) - a room equipped with physical items and devices that provide gentle multisensory stimulations through sounds, lights, projections, soft fabrics and materials.

Prior HCI research indicates that combining the physical and the digital world and offering multisensory stimuli through embodied interaction provides support for persons with NDD. Some works explored how tangible interaction with smart objects [3][9] smart toys [5], or soft robots [2] can help children with sensory processing disorders, particularly those with autism, to empower self-reflection, self-directed activity, and language. Several approaches exploit large displays and movement-based interaction. In the controlled study [1], the use of Kinect games in ASD therapy led to improvements in selective and sustained attention, visual perception, and visual-motor integration. SensoryPaint [8] [10] allows persons with NDD to paint on a large display using physical objects, movements and mid-air gestures, and to receive visual-aural stimuli. A field study showed that the use of the system balanced children's attention between their own bodies and sensory stimuli, and promoted socialization. MEDIATE [7] generates sound and visual stimuli in response to gestures and footsteps on the floor in front of a large display, and would stimulate creativity, sense of agency, and self-expression among low-functioning non-verbal children with autism.

\section{THE MAGIC ROOM}

The Magic Room is equipped with (Fig. 1-left) two projectors, one video camera, one ambient sound system, one Kinect, various smart objects (3D soft geometric shapes and stuffed toys), smart appliances (bubble maker and aroma emitter), mobile graspable smart lights, ambient lights, and a Philips Luminous Carpet ${ }^{\mathrm{TM}}$ (composed LED grids embedded in carpet tiles to enable the display of words and graphic patterns). The boards embedded in smart objects integrate pressure sensors, multi-axis accelerometers, gyroscope, magnetometer, and various actuators (for light, sound, and vibration). The Kinect detects mid-air gestures, body position, movements, and voice. A joystick enables caregivers to suspend/restart the current stimuli or activate new ones.

A multilayered software and hardware platform controls sensors, actuators and output devices, manages their communication, and orchestrates their behaviors. It integrates an interactive tool (called MAT - Fig. 1 right) to define the smart space behavior by associating stimuli to interactions or time events, and aggregating them. MAT has an intuitive visual interface that also nonICT specialists (e.g., caregivers) can easily learn.

The Magic Room combines and extends the features of existing multisensory digital systems in a unique way. In Sensory Paint and MEDIATE, stimuli and embodied interaction occur in front or in the immediate surroundings of the large display. In the Magic Room, these are pervasive in the entire physical environment. The children can move in the room and use the whole space to regulate the reactive behavior of interactive items. This would help them learn spatial awareness, i.e., the ability to be aware of oneself in space, to create a contextualized body schema representation, and to understand the spatial relationship between physical objects and oneself in the physical environment. Ambient sound and projections on the walls and on the floor, create a sense of immersion that is thought to be a powerful educational tool for subjects with NDD [11].

The Magic Room also offers stimuli inspired by Snoezelen such as lights, soap bubbles, and aromas. Differently from Snoezelen, these effects are created by "smart" devices connected to all room components, which enables to orchestrate stimulations in a countless number of ways. Smart lighting enables to create a vast gamut of luminous effects with different colors, intensity, and dynamics. In the Magic Room, lights neither provide pleasurable stimuli for relaxation or reward purposes like in Snoezelen, nor are merely embedded in tangibles [10]. They are pervasive in the physical environment, circumscribe the space, orient children's movements, and increase the immersive effect of the projections and the sense of magic. Mobile lights can be manipulated to offer focused luminous effects without introducing any other sensory stimuli.

Finally, the Magic Room considers that any technology for people with disability needs customization to address the unique characteristics of each person or group. While in existing smart multisensory systems, all features are "hard-coded" and any UX modification requires operations at programming level, using the simple visual tool MAT integrated of the Magic Room is very easy and better responds to customization needs. 


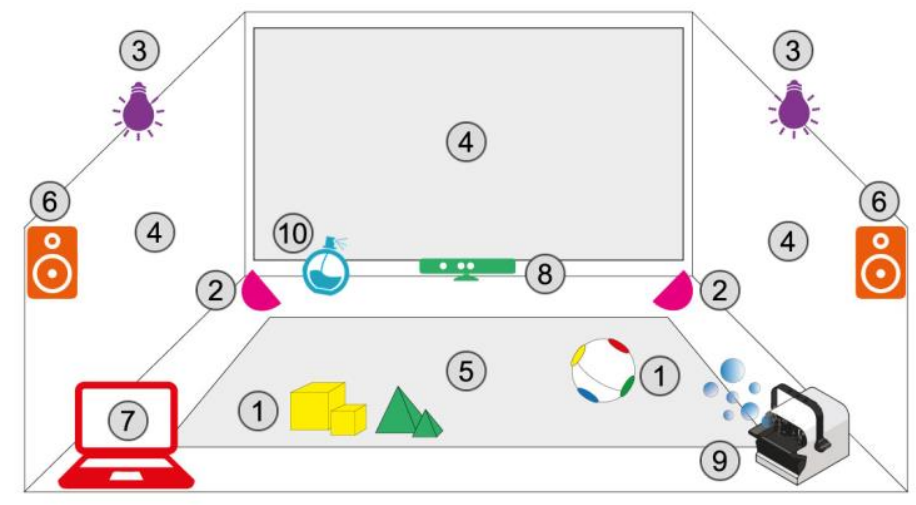

(1) Smart Objects and Toys (2) Mobile Graspable Lights; (3) Ambient Lights; (4) Wall Projection Area; (5) Floor Projection Area + Luminous Carpet; (6) Sound System; (7) PC; (8) Kinect; (9) Bubble machine, (10) Aroma Emitter

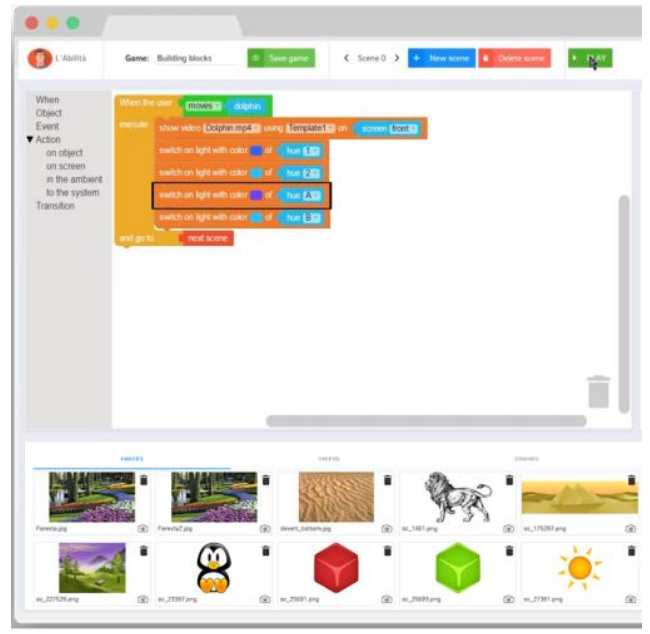

Fig. 1. The Magic Room: set-up (left) and MAT tool interface (right)

\section{DESIGNING THE CHILDREN'S EXPERIENCE}

We designed the children's experience in the Magic Room during three workshops (each one lasting for one weekend) held at the first care center where the Magic Room was deployed. We involved all therapists or special educators from the center (ten in total) and four groups of children with NDD who played in the Magic Room after workshop 1.

\section{Workshop 1}

After the caregivers had experienced the stimulatory and interaction capability of the Magic Room, we discussed how therapeutic programs, learning goals and practices at the center could inform the design of the children's experience in the smart space. We focused on free- play scenarios, initially using sketches and sticky notes, and then MAT to progressively prototype the coordinated behaviors of smart devices.

Supporting relaxation at the beginning of any experience in the smart space emerged as first requirement. Subjects with NDD tend to manifest anxiety in any change in routine. Helping children to reach a state of relaxation is fundamental to engage them in any experience. The caregivers proposed to use projections of videos employed for relaxation purposes in regular activities, and to integrate them with soft ambient lights of coordinated color.

We then defined scenarios for spontaneous interaction with smart toys (for example, the stuffed dolphin and the soft ball). When a sea world is projected and a child grasps the dolphin (and, in case of a verbal subjects, also says "blue"), the mobile lights turn on in blue; when the child releases the object, they turn off. These simple interactions would help children to familiarize with the smart environment and develop a sense of agency. Caregivers proposed to draw eyes and smile on the white fabric of the soft smart ball, to project an image that indicates a spatial relationship (e.g., a cat on the table), and to ask the child to press the corresponding area on the ball (e.g., "up"). In case of correct interaction, this area is highlighted, a nice ambient music is played, soap bubbles are emitted, and a happy face is projected. This simple form of play would promote visual-motor coordination and understanding of spatial relationships.

\section{Observing Children in the Magic Room}

We experimented the free play scenarios with the children attending the center. The exploratory nature of this experimentation required the inclusion of heterogenous subjects to understand how people with different capabilities might respond to an experience in the smart space. Therefore, we involved all children attending the center every afternoon and performed group activities that stimulate motor, social, and cognitive skills, lead to pleasure and wellbeing, and improve autonomous behavior. Each group was guided by two therapists and was composed of four to six children with similar educational needs and functional competences, regardless their diagnosis.

Children's profile is summarized in Table 1. "Severity" is a global measure of a child's impairments and is based on assessments performed twice a year that consider quantitative data from clinical tests (including IQ) and subjective qualitative observations by therapists and parents. "Severe" is normally associated to IQ=[30:35], "moderate" to IQ=[35:50] and "mild" to IQ= [50:70]. 
Each group used the Magic Room together with their two therapists for two or three sessions lasting in average 35 minutes. All sessions started with the projection of a relaxing environment integrated with soft lights. Eventually therapists introduced smart objects progressively, asking children to play with any item. All sessions were video-recorded. An intern therapist and a member of our team participated as observers.

Table 1: Profiles of the children participating in our research

\begin{tabular}{|c|c|c|c|c|c|}
\hline Gr. & $\begin{array}{c}\text { Child } \\
\text { ID }\end{array}$ & Gender & Age & Severity & Main Diagnosis \\
\hline \multirow{5}{*}{ G1 } & $\mathrm{C} 1$ & M & 8 & Mild & ASD (Autism Spectrum Disorder) \\
\hline & $\mathrm{C} 2$ & M & 8 & Moderate & ASD \\
\hline & $\mathrm{C} 3$ & M & 11 & Severe & Intellectual Disability + Bi-paresis \\
\hline & $\mathrm{C} 4$ & $\mathrm{~F}$ & 9 & Severe & Down Syndrome + ASD symptoms \\
\hline & $\mathrm{C} 5$ & $\mathrm{~F}$ & 10 & Severe & Down Syndrome + ASD symptoms \\
\hline \multirow{6}{*}{ G2 } & C6 & M & 9 & Mild & ASD \\
\hline & $\mathrm{C} 7$ & M & 9 & Moderate & Down Syndrome \\
\hline & $\mathrm{C} 8$ & M & 10 & Moderate & ASD \\
\hline & C9 & M & 12 & Moderate & ASD \\
\hline & $\mathrm{C} 10$ & M & 11 & Moderate & Intellectual Disability + Psychosis \\
\hline & $\mathrm{C} 11$ & $\mathrm{~F}$ & 11 & Severe & Down Syndrome + ASD symptoms \\
\hline \multirow{4}{*}{ G3 } & $\mathrm{C} 12$ & $\mathrm{~F}$ & 10 & Moderate & ASD \\
\hline & C13 & F & 8 & Mild & Prader Willi + ASD symptoms \\
\hline & C14 & M & 11 & Moderate & ASD \\
\hline & $\mathrm{C} 15$ & M & 8 & Mild & Prader Willi + Intellectual Disability \\
\hline \multirow{4}{*}{ G4 } & C16 & M & 10 & Moderate & ASD \\
\hline & $\mathrm{C} 17$ & M & 11 & Moderate & Intellectual Disability \\
\hline & $\mathrm{C} 18$ & M & 12 & Mild & ASD \\
\hline & $\mathrm{C} 19$ & M & 13 & Mild & Intellectual Disability \\
\hline
\end{tabular}

\section{Workshop 2}

We examined videos and observers' notes using deductive analysis based on the themes emerged in workshop 1. Several signals indicate that the experience was well accepted by all children. No child was afraid to enter the Magic Room nor experienced prolonged moments of distress. The time to reach a state of relaxation was different for each child but, according to caregivers, it was shorter than it normally happens when a change of routine occurs in the classroom. Some subjects (with mild or moderate impairment levels) manifested curiosity for the smart objects and manipulated them since the first session. Children with more severe disability remained seated quietly near the caregiver and did not interact with the smart space. Still, their eyes gaze was often directed towards active peers and the objects they were playing with. This is a positive result especially for children with autism. Two children who in the regular classroom were always in frantic movement, they progressively learned to control their excitement, wait for their turn, and sit still when requested by caregivers when in the Magic Room. Lights on the carpet and mobile lights as well as bubbles attracted the immediate attention of all subjects and suggest that these stimuli might have a special engagement power for children with NDD.

Before the experimentation, caregivers suspected that "children would get trapped in the projection like they do with TV". After the sessions, they concluded: "indeed this did not happen, children perceived the projection as part of a bigger environment that enhances not only their creativity but also the process of switching from imaginary worlds to the real one, and vice versa".

Caregivers regarded the opportunities for free play and the capability of smoothly repeating stimuli and interactions as useful to reassure the children and help them to consolidate cause-effects understanding. Still, caregivers also pinpointed the risk of boredom as well as the need for more challenging tasks and for activities focused on specific skills. We started to sketch new combinations of stimuli and interactions, which were elaborated in Workshop 3.

\section{Workshop 3}

This workshop was devoted to design "structured activities", exemplified below. These are experiences oriented towards specific goals that provide flows of "stimuli-interaction-stimuli" situations ("tasks") and require children to perform specific actions properly in order to proceed. We discussed the main learning goals addressed by regular interventions and incorporated the one that could be impacted by our smart environment.

"Shapes". This activity has cognitive learning goals and would help children understand simple concepts like shapes, sizes and colors. It is inspired by games performed in the regular classroom using colored blocks and colored chalk to draw on the floor. A color (or shape) is projected on the wall and luminous shapes appear on the carpet. Children are required to move on the carpet area matching the on-screen image, or to put their smart objects on the corresponding color or shape.

"Pass the light". This activity aims at practicing social skills, and is inspired by the classic Circle Game. The children pass a mobile light to one another after naming the light color, something with the same color, and the name of the child receiving the lamp. The lamp changes its color when shaken and changes color randomly. When it is red, a cheerful ambient music is played, bubbles appear, and game restarts. 
"Storytelling". In classroom, regular activities stories are used to convey information, exercise selective and sustained attention, motivate children in a task, and stimulate imitation skills by mimicking characters. In the Magic Room, a multimedia animated story is projected, pausing after each scene. To proceed to the next scene, children in turn must imitate the character(s) using gestures or movements, or to grasp colored smart objects that makes sense for the current scene. This may also trigger aromas and bubbles to enhance fun.

"Catch the...". This activity focuses on gross motor skills and was invented by caregivers without any reference to regular activities. A video is projected on the front wall (e.g., showing a galaxy with stars and planets) and luminous patterns alluding to the video (e.g., blinking white LEDs for the stars) randomly appear on the carpet. The children must jump on the patterns to make them disappear.
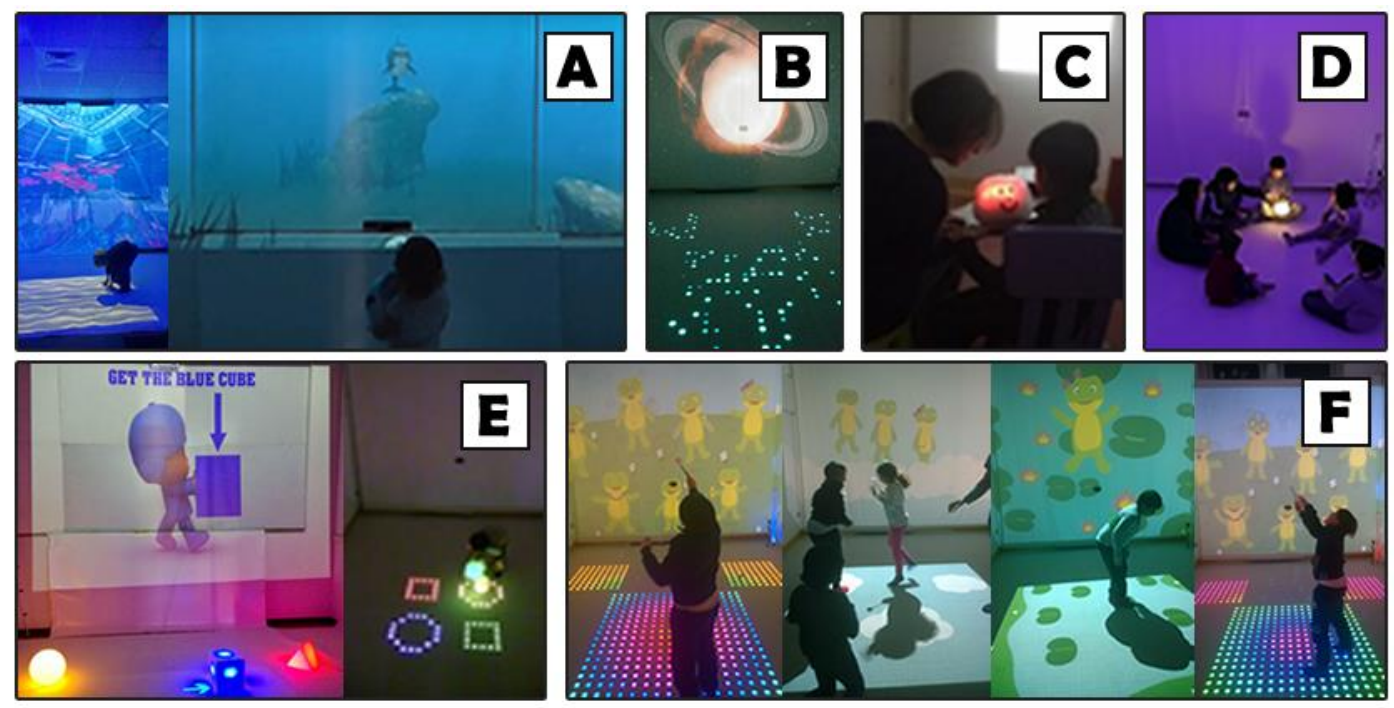

Fig. 2: Experiences in the Magic Room

A. Relaxation with sea-world projections, B. Catch the ..., C. Magic Ball, D. Pass the light, E. Shapes, F. Storytelling

\section{EXPLORATORY STUDY}

This study aimed at uncovering the Magic Room's potential to promote skills in specific areas, and involved all children who participated in the design process. Each group was planned to use the Magic Room with their two caregivers weekly for at least six sessions along three months. For each session, we defined an activity plan customized for each group. An intern therapist observed participants and took notes. All sessions were video-recorded.

Group caregivers and the observer (nine specialists in total) met at the end of each session and filled the observation form reported in Figure 3 for each child. The form is based on the main themes used in regular interventions and the learning goals addressed by the structured activities in the Magic Room. Goals are organized in macro-areas. They are associated to observable behaviors and a score that indicates the level of complexity of the associated behavior ( 1 is the lowest). A line is marked when the subject manifested the corresponding behavior(s) during the session. In the two months after the study caregivers kept a diary and annotated children's behaviors that, during regular activities, could refer to the experience in the smart space. 
Fig 3. Observation Form (left) and Main Quantitative Results (right)

\begin{tabular}{|c|c|c|c|}
\hline AREA & THERAPEUTICGOAL & BEHAVIOR & SCORE \\
\hline \multirow{7}{*}{$\begin{array}{l}\stackrel{\mathrm{Z}}{\mathrm{Z}} \\
\stackrel{\mathrm{Z}}{8}\end{array}$} & LONG TERM MEMORY L1 & $\begin{array}{l}\text { memory and/or recovery of only some } \\
\text { elements of the previous sessions }\end{array}$ & 1 \\
\hline & LONG TERM MEMORY L2 & $\begin{array}{l}\text { memory and/or recovery of almost all the } \\
\text { elements of previous sessions }\end{array}$ & 2 \\
\hline & CONCENTRATION L1 & keeps the focus for about $50 \%$ of the session & 1 \\
\hline & CONCENTRATION L2 & $\begin{array}{l}\text { keeps the focus for almost / the whole duration } \\
\text { of the session }\end{array}$ & 2 \\
\hline & SHORT TERM MEMORY LI & after three times doesn't perform the task & 1 \\
\hline & SHORT TERM MEMORY L2 & $\begin{array}{l}\text { after three times starts the action, but does not } \\
\text { perform the entire task }\end{array}$ & 2 \\
\hline & SHORT TERM MEMORY L3 & after three times properly executes the task & 3 \\
\hline \multirow{13}{*}{ 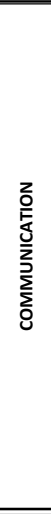 } & VERBAL MESSAGES RECEPTION & reacts to the human voice & 1 \\
\hline & VERBAL MESSAGES COMPREHENSION & understands simple verbal message & 2 \\
\hline & $\begin{array}{l}\text { VERBAL MESSAGES ADVANCED } \\
\text { COMPREHENSION }\end{array}$ & understands complex verbal message & 3 \\
\hline & NON-VERBAL MESSAGES RECEPTION & $\begin{array}{l}\text { reacts to body gestures and/or facial } \\
\text { movements }\end{array}$ & 1 \\
\hline & $\begin{array}{l}\text { NON-VERBAL MESSAGES } \\
\text { COMPREHENSION }\end{array}$ & understands simple non-verbal message & 2 \\
\hline & $\begin{array}{l}\text { NON-VERBAL MESSAGES ADVANCED } \\
\text { COMPREHENSION }\end{array}$ & understands complex non-verbal message & 3 \\
\hline & VERBAL MESSAGES PRODUCTION L1 & vocals / babbling & 1 \\
\hline & VERBAL MESSAGES PRODUCTION L2 & words & 2 \\
\hline & VERBAL MESSAGES PRODUCTION L3 & sentences & 3 \\
\hline & WILLINGNESS TO COMMUNICATE L1 & searches for the other & 1 \\
\hline & WILLINGNESS TO COMMUNICATE L2 & starts a conversation & 2 \\
\hline & WILLINGNESS TO COMMUNICATE L3 & starts, keeps and closes a conversation & 3 \\
\hline & ABSENCE OF ECHOLALIA & no automatic repetitions of vocalizations & 1 \\
\hline \multirow{8}{*}{ 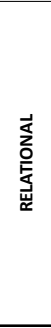 } & SOCIAL PREREQUISITES L1 & eye contact & 1 \\
\hline & SOCIAL PREREQUISITES L2 & shared attention & 2 \\
\hline & SOCIAL INTERACTION & turn-taking & 3 \\
\hline & SOCIAL PLAY L1 & alone play & 1 \\
\hline & SOCIAL PLAY L2 & shared play & 2 \\
\hline & SOCIAL PROXEMIC & mutual pleasure and relational skills & 1 \\
\hline & COLLABORATION L1 & $\begin{array}{l}\text { shows a moderately oppositional behaviour or } \\
\text { shows an inconstant collaboration }\end{array}$ & 1 \\
\hline & COLLABORATION L2 & $\begin{array}{l}\text { follows the educator's needs and try to work } \\
\text { together when they can least understand or do } \\
\text { what was required }\end{array}$ & 2 \\
\hline \multirow{2}{*}{  } & APPROPRIATENESS OF EMOTION L1 & $\begin{array}{l}\text { manifests a few appropriate emotions, with } \\
\text { little variation or moderately inappropriate }\end{array}$ & 1 \\
\hline & APPROPRIATENESS OF EMOTION L2 & manifests appropriate emotions & 2 \\
\hline \multirow{6}{*}{ 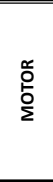 } & EYE-HAND COORDINATION L1 & critical / with help & 1 \\
\hline & EYE-HAND COORDINATION L2 & awkwardly & 2 \\
\hline & EYE-HAND COORDINATION L3 & appropriate & 3 \\
\hline & POSTURAL PASSAGES L1 & critical / with help & 1 \\
\hline & POSTURAL PASSAGES L2 & awkwardly & 2 \\
\hline & POSTURAL PASSAGES L3 & appropriate & 3 \\
\hline
\end{tabular}

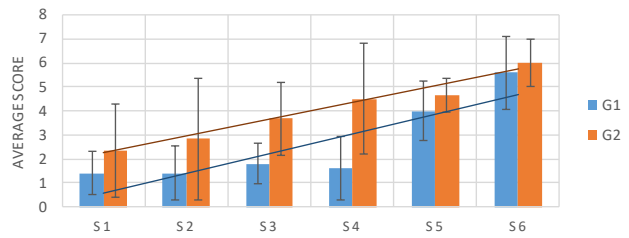

Diagram 1. Avg. per Group - Cognitive Area

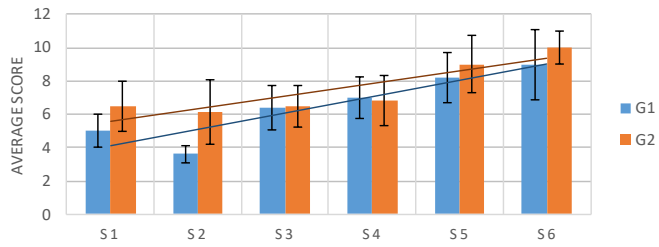

Diagram 2. Avg. per Group - Communication Area

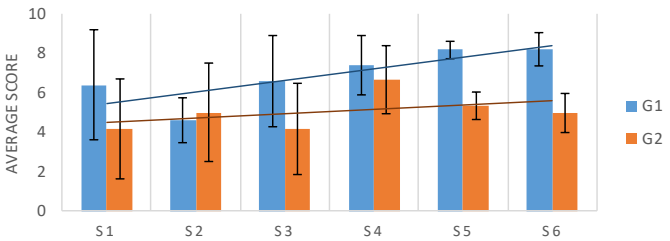

Diagram 3. Avg. per Group - Relational Area

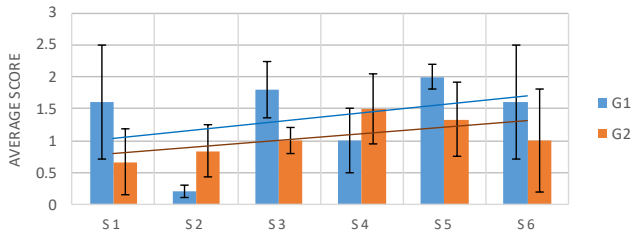

Diagram 4. Avg. per Group - Emotional Area

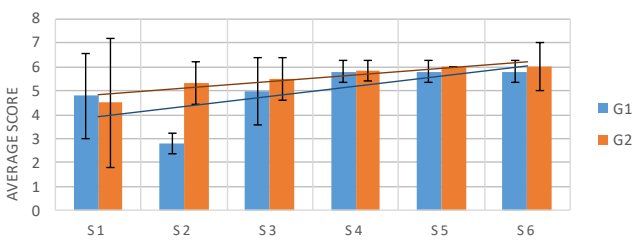

Diagram 5. Avg. per Group - Motor Area

\section{Results}

The original activity plan was continuously adapted during the study to meet the evolving needs of children. Some activities were sometimes repeated, within and across sessions, particularly those that the children explicitly requested. All members of Group 1 (G1) and Group 2 (G2) attended all sessions. For various reasons (e.g., health conditions) several children from the other groups participated in two or three sessions only. The session duration progressively increased, lasting in average 39' (min. 20'- max. 52').

We performed data analysis with all specialists at the center following a mixed-method approach. To analyze qualitative data (videos and textual notes in the form and the diary), we used Grounded Theory techniques grouping quotes or events to uncover emerging themes related to children's behaviors. For each session, we aggregated quantitative data from the forms by child, summing the scores for all behaviors that were marked by caregivers for that child, and by group of children.

For lack of space we discuss only the main results. Figure 3 (right) plots by session and by area the average value on all children in the same group, considering G1 and G2 who attended all sessions. Diagrams also show SD (standard deviation) per child and trends across sessions (cross-bar lines).

As it happens in most studies in the NDD domain, SD pinpoints a strong variability among subjects. Still, the comparison between the first and last sessions and the trends might indicate improvements of both groups in all areas. 


\section{Cognitive Area}

In this area caregivers observed the strongest improvements. The values in the last session are almost the double than the initial ones for G2 and the triple for G1. The caregivers detected several positive signals in this area during storytelling activities. A therapist annotated: "children started to pay attention to the story more quickly than usual and sustained attention for a longer time... they were living those moments inside the story in first person". We observed the persistency of some positive results related to memory that surprised the therapists. During the study period, and in the following weeks, all children, verbally or gesturally, pointed to the room when passing by. Several children, including those with severe memory deficits and difficulties in externalizing needs, asked to go to the room again and play their favorite games. Caregivers noticed signals of generalization for concepts related to stories. C2 built associations between the story experience in the Magic Room and events happening in the classroom, saying "like in Piera the Frog story?"

\section{Communication and Relational Areas}

Comparing the first and the last sessions, G1 showed an increment of $28 \%$ in the relational area and $64 \%$ in communication. The latter was mainly associated to improved verbal skills and intentional communication. C5 did not communicate spontaneously with peers and caregivers. The second time she played with the smart dolphin, she started speaking with the fishes in the projection and eventually also with therapists and mates ("Look Mario at that big fish! ...moving away!"). In the last session, a child who had problems in formulating words (C3) could spell colors correctly (saying "rosso", the Italian for "red", instead of "re..re" or "rozz"). Storytelling activities seemed to be particularly effective to trigger socialization behaviors. A usually shy and passive girl (C13) started manifesting pro-active behaviors towards her mates never manifested before, inviting them to play together ("Let's use the magic stick together like Piera the frog!".) C12, who seldom externalizes curiosity, started asking the caregiver "What's happening next?" during a story pause.

\section{Emotional Area}

The comparison between the first and the last sessions shows an increase of $25 \%$ for G1 and $80 \%$ for G2. One child (C8) who very seldom externalized emotions, in the last session smiled and applauded his-self after completing the "Shape" activity. Some children perceived the room as a magic space, and light effects seemed to be particularly effective to promote this feeling. "Today I went to a place, if I were to say blue, everything would have become blue like the sea [because the ambient blue light turned on] ... and there is a dolphin you can touch and then it shows a rainbow of light on its back! We were doing enchantments!" (C5 talking with his parents).

\section{Motor area.}

Here the observed improvements were weaker than in other areas for G1 (+20\%) and in the average (+ 33\%) for G2. Still, according to caregivers, most children demonstrated a much stronger attitude to movement than in regular activities. "Some children...engaged in motor activities that are complex for them without even noticing they were pushing harder, overcoming their own limits, and doing movements we've never ever seen them doing”. For example, a child with biparesis (C3) completed a motor task that required her to reach the upright posture and to move and walk, and she did so without asking for help.

\section{Problematic behaviors}

We observed also some negative behaviors that needed to be regulated by caregivers. For example, C8 became over excited and hyperactive by effects of the bubbles emitted as reward after a task during the first session; he pushed away the other children to hit as many bubbles as possible, and at the end started running frantically in the room until the caregivers grasped him and helped him to reassess and stabilize himself. While waiting for his turn during a storytelling activity, C7 started kicking the Magic Ball and became aggressive toward peers. The therapists annotated "The pleasure resulting from the experience may have created excitement and an intensive willingness to play; this can be frustrating and make self-regulation and control more difficult. In addition, while waiting for their turn children feel in "competition" with peers for the appropriation of the play stage and of the therapist's attention".

\section{Conclusions}

To our knowledge, the Magic Room is unique in that it supports a gamut of stimuli, interaction modes, and play experiences that are not available all together in existing smart spaces for children with NDD or in the Snoezelen. The Magic Room could be integrated in regular practices at a relatively affordable installation cost (less than 1/4 of commercial Snoezelen, which requires 30.000 euros per installation and is adopted in many schools and therapeutic centers in US, Canada, and Australia). A Magic Room can be regarded as a living lab to enable the exploration of how children with NDD behave when exposed to different controlled stimuli and interaction modes and the experimentation of innovative scenarios of intervention.

Our exploratory study exemplifies how children with NDD and their caregivers might use the Magic Room to develop a variety of skills. Approximately every two months after the end of the study, we have interviewed the caregivers using the smart space on a regular base; interesting insights and new uses of the smart space are continuously emerging. The empirical results of the 
exploratory study are encouraging. According to caregivers' subjective judgment, the learning effects observed in the study were achieved much faster compared to traditional interventions in the classroom. Still, our findings are very preliminary and should be taken with great caution. Our study has many limitations, mainly due to the lack of control on several variables as well as the variability between the subjects. Missing a control group, we cannot claim that the children's improvements are ascribed to the Magic Room's use as opposed to just general growth associated to each individual's development or to other experiences outside the smart space.

The next step in our research agenda is to perform a more rigorous empirical study for one year at the care center where the second Magic Room is installed. We will involve 60 subjects and complement descriptive results with clinical tests performed before, during, and after the study, to compare the effects of the Magic Room against regular interventions and against treatments in a Snoezelen.

\section{References}

1. Bartoli L. et al. Designing and evaluating touchless playful interaction for ASD children. In Proceedings of the 2014 Conference Interaction Design and Children (IDC '14). ACM, 17-26.

2. Bonarini, A. et al., A huggable, mobile robot for developmental disorder interventions in a multi-modal interaction space. IEEE ROMAN 2015, 823-830

3. Escobedo, L . et al. Smart objects to support the discrimination training of children with autism. Personal and Ubiquitous Computing, 18 (6) 2014, 1485-1497

4. Fahle, M. and Poggio, T. eds., 2002. Perceptual learning. MIT Press.

5. Hengeveld, B., Voort, R., Hummels, C., de Moor, J., van Balkom, H., Overbeeke, K. and van der Helm, A., 2008. The development of LinguaBytes: an interactive tangible play and learning system to stimulate the language development of toddlers with multiple disabilities. Advances in Human-Computer Interaction, 2008, p.1.

6. Lancioni, G.E., Cuvo, A.J. and O'Reilly, M.F., 2002. Snoezelen: an overview of research with people with developmental disabilities and dementia. Disability and rehabilitation, 24(4), pp.175-184.

7. Pares, N., et al. 2005. Achieving dialogue with children with severe autism in an adaptive multisensory interaction: the" MEDIATE" project. IEEE Transactions on Visualization and Computer Graphics, 11(6), pp.734-743

8. Ringland, K.E., Zalapa, R., Neal, M., Escobedo, L., Tentori, M. and Hayes, G.R., 2014, September. SensoryPaint: a multimodal sensory intervention for children with neurodevelopmental disorders. In Proceedings of the 2014 ACM International Joint Conference on Pervasive and Ubiquitous Computing (pp. 873-884). ACM.

9. Tam, V., Gelsomini, M. and Garzotto, F., 2017, March. Polipo: a Tangible Toy for Children with Neurodevelopmental Disorders. In Proceedings of the Tenth International Conference on Tangible, Embedded, and Embodied Interaction (pp. 11-20). ACM.

10. Tentori, M., Escobedo, L. and Balderas, G., 2015. A smart environment for children with autism. IEEE Pervasive Computing, 14(2), pp.42-50.

11. Wallace, S., Parsons, S., Westbury, A., White, K., White, K. and Bailey, A., 2010. Sense of presence and atypical social judgments in immersive virtual environments: Responses of adolescents with Autism Spectrum Disorders. Autism, 14(3), pp.199-213.

12. Wilson, M. Six views of embodied cognition. Psychonomic Bulletin \& Review, 9, 625-636. Springer-Verlag, 2002 